\title{
Location-Based Services in a Smartphone
}

\author{
Rakesh I. Aralikatti and Kishan S. Anegundi
}

\begin{abstract}
The aim of this research is to give promote knowledge into conceivable outcomes to PDA cutting edge Location Based Services (LBS) applications, including a more noteworthy comprehension of usage and design contemplations, and future patterns in LBS. This report traces the ebb and flow condition of LBS innovation, inquire about into the vital issues of keeping up client security, and future patterns, for example, enhanced perception through enlarged reality and moves towards connection based registering and Semantic Web. What's more, more critically to plan and compose Android Application to make "Customary neighborhood news media". Customary neighborhood news media: It is like nearby news coverage beginning from a little city and extending this application to reach to a national level to cover whole nation. We begin with the neighborhood daily paper's plan of action all the while. The online networking grabs the tattles from your group of friends and intrigue bunches, pulling perusers' consideration far from the printed page. Safe to say, it's been something of an immaculate tempest misusing customary neighborhood news media. Alongside which the entrance of this is made through the cellps for the cell phone.
\end{abstract}

Keywords--- Location Based Services, Local News Media, Social Circle, Interested Groups, Location Determining Technologies.

\section{INTRODUCTION}

A Location-Based Service (LBS) is a mobile computing application that provides information and functionality to users based on their geographical location. First generation examples include - show me nearby restaurantll-type applications.

Next-generation LBS can provide additional benefits for users and service providers, including:

1. Proactively pushing only relevant information to users to help speed up decisions and activities.

2. Minimizing tedious data entry by integrating applications with advanced sensors such as accelerometers, digital compasses and cameras.

3. Allowing service providers to model user behavior based on their location and route information, which can support improvements of service levels in near real-time and over the longer term.

Rakesh I. Aralikatti, Student, Dept., of Computer Science and Engineering, Sksvmacet-laxmeshwar.E-mail: rakeskiaralikatti@gmail.com

Kishan S. Anegundi, Student, Dept., of Computer Science and Engineering, Sksvmacet-laxmeshwar.E-mail:anegundikishan@gmail.com DOI:10.9756/BIJSESC.8259
4. Encouraging sharing of location-based information, such as photos and reviews, generated by other service providers and users.

Global Positioning System (GPS) is widely used for the Location-Based Service (LBS) of smartphones. However, GPS dramatically increases the energy consumption of a smartphone due to heavy computation overhead [1-10]. Specifically, a GPS receiver in a smartphone polls signals from multiple satellites continuously and calculates the threedimensional location (latitude, longitude, and altitude), which increases the energy consumption of a smartphone dramatically [12]. It is reported that the battery life of a smartphone is less than 12 hours when GPS is turned on although no other activity is performed on the smartphone [1, $3-5,13]$. This is a serious problem as it prevents smartphone users from continuing their activities when the battery is exhausted. To relieve this problem, some alternative positioning techniques for smartphones have been studied [310, 14-19]. Cell-PS (Cell-ID Positioning Scheme) is one of the representative techniques used for the smartphone positioning instead of GPS. As smartphones continuously receive the Location Area Identity (LAI) and cell-id broadcast by Base Transceiver Stations (BTSs), Cell-PS uses this information for the positioning of a smartphone [16, 22, 23]. Cell-PS is energy-efficient and it can also be utilized indoors [8, 9, 23]. However, the accuracy of Cell-PS is not satisfactory compared to GPS [28].

WiFi Positioning System (WiFi-PS) is another technique that can be used instead of GPS. WiFi-PS uses the Media Access Control (MAC) address of WiFi access points and delivers the address to the server for positioning [18]. The accuracy of WiFi-PS depends on the number of locations stored in the database and the access point density [16, 19]. The variations of signals can cause large error and the maximum error would be around 1,000 meters [19]. Another weakness of WiFi-PS is that it can be adopted only in a certain limited area. Some smartphone sensors such as accelerometer sensor or orientation sensor can also be used for the positioning of smartphones. However, sensors consume additional energy and their accuracy is not as good as that of GPS.

\section{DETAILED OUTLINE}

We list and give details of various items considered here.

Aim of the research - Local News Distribution Centre We start with the design of a small local city news gathering and segregating into various categories. We will discuss various options to find out exact location of a user be on a block and street. There has never been so many sources of local news available, given the web has removed barriers to publishing, social media has got everyone accustomed to self-publishing (and self-publicizing), and smart phones are individual e- 
readers in everyone's pockets. Local news is out there and people are interested in reading stuff, so distributing relevant content to the right people is one new challenge. The focus is on giving local news to a small town. This city-based startup is aggregating local news sources, from blogs eventually to newspapers distributed in big city and finally reaching to local location like a particular street or block where someone with smart phone is going around. Our front page uses social activity as part of how we prioritize things which is our primary way of displaying information to the user. If a news item is commonly available on different social media sites, it means the news item is quite important to be added in local database. Figure 1 shows the path of flow of local news taken from different sources and taking it to the mobile user.

We have been able to develop such an application in android. We have discovered that 1100 users used this site for an average of 30 to 45 minutes per day in first one week. Advantage is we did not need any marketing. The next job is to launch similar somewhat modified application in some bigger cities and in a few months do it for a very large city and eventually repeat it at country level.

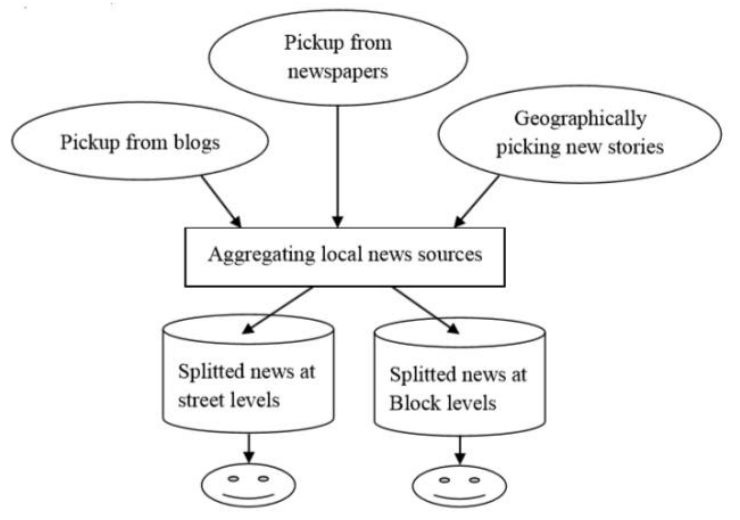

Figure 1: Designing and Distributions of Small City Local

\section{Newspaper for Mobile User}

Another Advantage is that you don't have to be journalism professional to be associated with this system. The startup is not purely technology driven. It is currently employing one human to keep an eye over the news stories and deciding on whether stories should be included. The categorization of news as per the location is derived using some machine learning algorithm. There's an attempt to extract location data. In this case one editor is able to handle that entire set of articles rather than same work is done by several editors for editing news articles of a large newspaper company.

\section{A) Local News at A Glance}

Moreover, while locally motivated bloggers may be keen to cover certain types of local news, say like a new ice cream shop opening in some block, other less easy to report one community goings-on may well fall through the cracks of a citizen journalism, crowd sourced news model. We looked at the technology that came in this field over the past few years. Location-based services (LBS) have been using GIS. A location-based service can be defined as an information service provided by a device that knows where it is, and modifies the information it provides accordingly. GPS integrated with a database in a mobile device such as a cell phone can provide a powerful form of enhanced reality. A more dynamic approach results when the mobile device is linked wirelessly to the internet, allowing more-or-less continuous updating. Information on minute-by-minute changes in traffic congestion, the positions of trains and buses, and the realtime locations of flights can be continuously fed from servers, providing truly dynamic LBS. For example, due to use of GPS, it is now possible to obtain real-time position of any train is determined and hence informs the user the time it will take more to reach an already declared railway station. Several Web services offer realtime data on traffic densities on major roads and several airlines allow users to query the locations of flights, returning the results in map form.

An emergency responder may find it difficult to determine his or her current location, particularly if street signs and house numbers have been found to be not visible, or if the street pattern itself has become distorted, or if visibility is obscured by smoke or dust. In such situations it is useful to have a mobile device equipped with GPS and a map database. Several cell phone operators now provide variations on the theme of mapping friends. For example, the Find Friends service offered in some markets by AT\&T. Tracking devices monitor the location of the device on a fixed interval of time. Often the major constraint on the frequency of sampling and the length of time over which data can be collected is the capacity of batteries, and this is particularly problematic when devices have to be carried by small mammals or birds. But when battery power is not a constraint, for example when tracking devices are carried on vehicles, it is common for sampling intervals to be as short as 1 second. With such rates it is possible to detect stops, starts, and changes of speed, and thus to infer many potentially useful properties.

\section{B) Location Determining Technologies}

GPS requires the line-of-sight presence of at least three satellites for horizontal positioning, and at least four for additional vertical positioning. Unfortunately this means that GPS signal is frequently lost by mobile devices, due to tree cover, steep slopes, and buildings - and is never available within buildings. For pedestrians and for vehicles parked in structures the percentages are substantially lower.

Many options for filling these coverage gaps in positioning technology have been explored, especially within buildings, with varying degrees of success. Beacons can be installed at fixed positions, radiating signals in various parts of the spectrum that can be used by mobile devices to determine position (Wi-Fi signals offer one of the more successful options ). At this time, however, no one system appears to be ideal, and to offer smooth interoperability and transition with GPS positioning outdoors. Meanwhile research projects continue to develop prototypes of integrated indoor/outdoor positioning. Meanwhile research projects continue to develop prototypes of integrated indoor/outdoor positioning. RFID tags are now being used by major retailers to keep track of the production, shipment, storage, and sale of goods.

In this paper, we present a new positioning scheme for 
smartphones, called EEPS (Energy-Efficient Positioning Scheme). EEPS adaptively performs the positioning of a smartphone considering the interesting regions of a user, accuracy requirement of each application executed within the smartphone, and the battery level, in order to minimize the energy consumption of a smartphone. In particular, EEPS predetermines the interesting regions of a user, which refer to the locations that the user stayed for a certain period of time like home, school, or workplace. If the user is in the interesting regions, EEPS can use Cell-PS instead of GPS to reduce energy consumption. EEPS also takes different actions according to the positioning accuracy required by the applications executed and the remaining battery level of the smartphone. Specifically, EEPS categorizes applications according to the required level of positioning accuracy, and then adaptively utilizes GPS and Cell-PS. To evaluate the effectiveness of EEPS, we perform simulations under four location based applications and five scenarios. The evaluation results show that EEPS reduces the energy consumption of GPS by $50.1 \%$ on average. Nevertheless, the error of EEPS is within an acceptable range, thereby meeting the requirement of each application. Table I briefly compares the characteristics of different positioning techniques.

Table 1: Comparison of Positioning Schemes

\begin{tabular}{|l|l|l|l|}
\hline & \multicolumn{1}{|c|}{ GPS } & \multicolumn{1}{c|}{ WiFi-PS } & \multicolumn{1}{c|}{ Cell-PS } \\
\hline $\begin{array}{l}\text { Energy } \\
\text { consumption }\end{array}$ & VERY & MEDIUM & LOW \\
\hline Accuracy & HIGH & LOW & LOW \\
\hline Availability & OUTDOORS & IN/OUTDOORS & IN/OUTDOORS \\
\hline $\begin{array}{l}\text { Response } \\
\text { Time }\end{array}$ & SLOW & MEDIUM & FAST \\
\hline
\end{tabular}

C) Social Issues

It will be apparent already that many forms of LBS raise serious and significant issues of an ethical nature, whether or not such access has been explicitly authorized. From the service provider, the _app" is meant to help the public. Many of the applications allow third parties to gain access to information about the location of the user,

1) To a public safety answering point, emergency medical service provider or emergency dispatch provider, public safety, fire service, or law enforcement official, or hospital emergency or trauma care facility, in order to respond to the user's call for emergency services;

2) To inform the user's legal guardian or members of the user's immediate family of the user's location in an emergency situation that involves the risk of death or serious physical harm; or

3) To providers of information or database management services solely for purposes of assisting in the delivery of emergency services in response to an emergency."

\section{RESEARCH MODEL}

Operational Definition and Survey Items of the Research Constructs: The research constructs used in this study were measured using survey item scales with confirmed reliability and validity from previous studies. These measures were modified to fit the LBS context. In a recent study on the actual use of mobile web browsing services, correlation analyses among variables related to usage behavior were conducted, proving that usage frequency represents usage behavior very well. Therefore, actual usage behavior is measured as the usage frequency of LBS in this study. Performance expectancy is defined as the degree to which one believes that the use of a certain technology will be useful for enhancing task performance. Performance expectancy will positively affect the continuous usage intention of LBS. Effort expectancy in the LBS context, relates to an individual's expectation of being able to use LBS to obtain necessary information or services without exerting much effort or encountering much difficulty. Effort expectancy will positively affect the continuous usage intention of LBS. Social influence can be defined as the degree to which an individual believes that he or she is expected to use the technology.

\section{LBS ARCHITECTURES AND PLATFORMS}

A notable characteristic is that the system can provide the information in Wireless Application Protocol (WAP) specified standards. This means the system can provide services to a wider range of users. The authors have discussed a component based architecture where different components are responsible for different functions. These components interact to detect the users, to estimate their location, to authenticate them and to provide them location related data.

Case Study: Such a design based on modular architecture gives some useful insight into how complex campus information systems can be managed in an easy way. One can describe how guests or visitors can receive information regarding the surrounding environment. This is important since visitors may visit campus for conferences, seminars and other purposes. Voice XML or VXML technology allows a user to interact with the Internet through voice-recognition technology. Using VXML, the user interacts with voice browser by listening to audio output that is either pre-recorded or computer synthesized. They can provide audio input through voice or through a keypad, such as that of mobile phones. This provides an easier way for the users to query the system for information. Moreover this system will incorporate even a wider community of users receiving LBS because the user does not need a smart phone to get these services. A low cost mobile phone would be enough. However VXML on the other hand will require some other associated functions and components like accurate speech recognition and VXML platform. The delay and bandwidth requirements for VXML applications are also major issues to be investigated.

\section{CONCLUSIONS}

We have provided context-based computing and Semantic Web. We have designed and written Android Application to make - Traditional local news media. Local news was generated and displayed on a user's mobile screen. Mobile's details of location of block and street are also taken in whereas so far only location of a major road is shown by GPS. We have deeply studied some future products in the Indoor 
Wireless Network, VXML, it's Mapping-Quest, Earth mine For 3D Street Level Imaging and, automated data processing pipelines, cloud based hosting services and server software, to desktop software, client side developer tools and direct integration

with

GIS software.

\section{REFERENCES}

[1] H. Yun, D. Han and C.C. Lee, "Understanding the use of location based service applications: Do privacy concerns matter", Journal of Electronic Commerce Research, Vol. 14, No. 3, 2013.

[2] C.W. Tan, M.S.Z. Khan, T. Silvadorai, T. Anwar and S. Ramadass, "A Glimpse into the Research Space of Location Based Services", Journal of Advances in Information technology, Vol. 3, No. 2, Pp. 91-106, 2012.

[3] J. Baek, S. Park and C.C. Lee, "Identifying Drivers for Continual Usage of Wireless Broadband", International Journal of Mobile Communications, Vol. 9, No. 4, Pp. 317-340, 2011.

[4] S.E. Hill and D.M. Buss, "Risk and Relative Social Rank: Positional Concerns and Risky Shifts in Probabilistic Decision-Making", Evolution and Human Behavior, Vol. 31, No. 3, Pp. 219-226, 2010.

[5] A. Küpper, "Location-Based Services: Fundamentals and Operation", New York: Wiley, 2005.

[6] J. Dillemuth, "Map design for mobile display", Proceedings, Auto Carto 2005.

[7] J.E. Dobson and P.F. Fisher, "Geoslavery", IEEE Technology and Society Magazine, Vol. 22, No. 1, Pp. 47-52, 2003.

[8] V. Nidhi, "Determining the Algorithm for Location Based Services Using Indoor Positioning Techniques", International Journal of Advanced Research in Computer Science and Software Engineering, Vol. 2, No. 7, 2012.

[9] G.P. Perrucci, F.H. Fitzek and J. Widmer, "Survey on energy consumption entities on the smartphone platform," In Conference on Proceedings of the 73rd Vehicular Technology, Pp. 1-6, 2011.

[10] A. Carroll and G. Heiser, "An analysis of power consumption in a smartphone", Proceedings of the USENIX Annual Technical Conference, 2010, pp. 271-284.

[11] Y. Chon, E. Talipov, H. Shin, H. Cha, "Mobility prediction-based smartphone energy optimization for everyday location monitoring", Proceedings of the 9th ACM Conference on Embedded Networked Sensor Systems, Pp. 82-95, 2011.

[12] J. Paek, J. Kim and R. Govindan, "Energy-efficient rate-adaptive GPSbased positioning for smartphones", Proceedings of the 8th International Conference on Mobile Systems, Applications, and Services, Pp. 299-314, 2010.

[13] Z. Zhuang, K.H. Kim and J.P. Singh, "Improving energy efficiency of location sensing on smartphones", Proceedings of the 8th International Conference on Mobile Systems, Applications, and Services, Pp. 315-330, 2010.

[14] M.B. Kjaergaard, J. Langdal, T. Godsk and T. Toftkjaer, "EnTracked: energy-efficient robust position tracking for mobile devices", Proceedings of the 7th International Conference on Mobile Systems, Applications, and Services, Pp. 221-234, 2009.

[15] Y. Wang, J. Lin and M. Annavaram, "A framework of energy efficient mobile sensing for automatic user state recognition", Proceedings of the 7th International Conference on Mobile Systems, Applications, and Services, Pp. 179-192, 2009.

[16] J. Paek, K. Kim, J.P. Singh and R. Govindan, "Energy-efficient positioning for smartphones using cell-ID sequence matching", Proceedings of the 9th International Conference on Mobile Systems, Applications, and Services, Pp. 293-306, 2011.

[17] N. Deblauwe and P. Ruppel, "Combining GPS and GSM cell-ID positioning for proactive location-based services", Proceedings of the 4th Annual International Conference on Mobile and Ubiquitous Systems: Computing, Networking and Services, Pp. 1-7, 2007.

[18] M.B. Kjærgaard, "Location-based services on mobile phones: minimizing power consumption", IEEE Pervasive Computing, Vol. 11, No. 1, Pp. 67-73, 2012.

[19] J. Liu, B. Priyantha, T. Hart, H. Ramos, A.A.F. Loureiro and Q. Wang, "Energy efficient GPS sensing with cloud offloading", Proceedings ACM Conference on Embedded Networked Sensor Systems, Pp. 85-98, 2012.
[20] F. Abdesslem, A. Phillips and T. Henderson, "Less is more: energy efficient mobile sensing with Sense Less", Proceedings of the 1st ACM SIGCOMM workshop on Networking, Systems, and Applications for Mobile Handhelds, 2009, Pp. 61-62.

[21] J. Georgy, A. Noureldin and C. Goodall, "Vehicle navigator using a mixture particle filter for inertial sensors/odometer/map data/GPS integration”, IEEE Transactions on Consumer Electronics, Vol. 58, No. 22, Pp. 544-552, 2012.

[22] Y. Chon, W. Ryu and H. Cha, "Predicting smartphone battery usage using cell tower ID monitoring”, Pervasive and Mobile Computing, Vol. 13, Pp. 99-110, 2014.

[23] K. Lin, A. Kansal, D. Lymberopoulos and F. Zhao, "Energy-accuracy trade-off for continuous mobile device location", Proceedings of the International Conference on Mobile Systems, Applications, and Services, 2010.

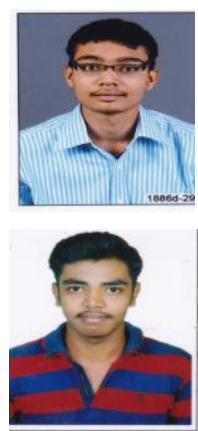

Rakesh I. Aralikatti, Sksvmacet-laxmeshwar, India. (E-mail:rakeskiaralikatti@gmail.com)

Kishan S. Anegundi,

Sksvmacet-laxmeshwar, India. (E-mail):anegundikishan@gmail.com 